\title{
Effect of pressure on the steplike magnetostriction of single crystalline bilayered manganite $\left(\mathrm{La}_{0.4} \operatorname{Pr}_{0.6}\right)_{1.2} \mathrm{Sr}_{1.8} \mathrm{Mn}_{2} \mathrm{O}_{7}$
}

\author{
Y. Yamato, ${ }^{1}$ M. Matsukawa, ${ }^{1, *}$ T. Kumagai,${ }^{1}$ R. Suryanarayanan, ${ }^{2}$ S. Nimori,${ }^{3}$ M. Apostu, ${ }^{4}$ A. Revcolevschi, ${ }^{2}$ K. Koyama, ${ }^{5}$ \\ and N. Kobayashi ${ }^{5}$ \\ ${ }^{1}$ Department of Materials Science and Engineering, Iwate University, Morioka 020-8551, Japan \\ ${ }^{2}$ Laboratoire de Physico-Chimie de L'Etat Solide, CNRS, UMR8182, Universite Paris-Sud, 91405 Orsay, France \\ ${ }^{3}$ National Institute for Materials Science, Tsukuba 305-0047, Japan \\ ${ }^{4}$ Department of Physical, Theoretical and Materials Chemistry, Faculty of Chemistry, Al. I. Cuza University, \\ Carol I, 700506 Iasi, Romania \\ ${ }^{5}$ Institute for Materials Research, Tohoku University, Sendai 980-8577, Japan
}

(Received 2 July 2008; revised manuscript received 4 September 2008; published 29 October 2008)

\begin{abstract}
We report the effect of pressure on the steplike magnetostriction of single crystalline bilayered manganite $\left(\mathrm{La}_{0.4} \mathrm{Pr}_{0.6}\right)_{1.2} \mathrm{Sr}_{1.8} \mathrm{Mn}_{2} \mathrm{O}_{7}$, for our understanding of the ultrasharp nature of the field-induced first-order transition from a paramagnetic insulator to a ferromagnetic metal phase. The application of pressure suppresses a steplike transformation and causes a broad change in the magnetostriction. The injection of an electric current to the crystal also weakens the steplike variation in both the magnetostriction and magnetoresistance. The stabilization of ferromagnetic interaction or the delocalization of charge carriers is promoted with the applied pressure or applied current, resulting in the suppressed steplike behavior. Our findings suggest that the step phenomenon is closely related to the existence of localized carriers such as the short-range charge-orbitalordered clusters.
\end{abstract}

DOI: 10.1103/PhysRevB.78.132411

\section{INTRODUCTION}

Perovskite manganites show a great variety of fascinating properties such as colossal magnetoresistance (CMR) effect and charge-ordered insulating phase. ${ }^{1}$ The most interesting one is the existence of a phase-separated state, the coexistence of antiferromagnetic charge-orbital-ordered (COO) insulating and ferromagnetic (FM) metal regions. ${ }^{2}$ Several recent studies on metamagnetic transition in phase-separated manganites have revealed that ultrasharp steps in magnetization curves appear at low temperatures. ${ }^{3-8}$ To account for this, a martensitic model due to local strain fields stored in the lattice between competing COO and FM phases with their different unit cells has been proposed though questions have been raised against this model.

For our understanding of the dynamics of a steplike firstorder transition from a paramagnetic insulating (PMI) to a ferromagnetic metal (FMM) phase in CMR manganites, we examine the pressure effect on $c$-axis magnetostriction of single crystalline $\left(\mathrm{La}_{0.4} \mathrm{Pr}_{0.6}\right)_{1.2} \mathrm{Sr}_{1.8} \mathrm{Mn}_{2} \mathrm{O}_{7}$. For the Prsubstituted $\left(\mathrm{La}_{0.4} \mathrm{Pr}_{0.6}\right)_{1.2} \mathrm{Sr}_{1.8} \mathrm{Mn}_{2} \mathrm{O}_{7}$ crystal, a spontaneous ferromagnetic metal phase (originally present with no $\mathrm{Pr}$ substitution) disappears at the ground state but a fieldinduced PMI to FMM transformation is observed over a wide range of temperatures. ${ }^{9,10}$ A magnetic $(H, T)$ phase diagram, established from magnetic measurements, is separated into three regions labeled as PMI, FMM, and bistable states, as shown in Fig. 1 of Ref. 11. The proximity of free energies between the PMI and FMM states is of importance to realize the bistable state where the FMM and PMI states coexist. A stronger field is needed to induce the PMI to FMM transition at low temperatures since the thermal energy is reduced upon lowering temperature.

\section{EXPERIMENT}

Single crystals of $\left(\mathrm{La}_{1-z}, \mathrm{Pr}_{z}\right)_{1.2} \mathrm{Sr}_{1.8} \mathrm{Mn}_{2} \mathrm{O}_{7}$ ( $\left.z=0.6\right)$ were grown by the floating-zone method using a mirror furnace.
PACS number(s): $75.80 .+\mathrm{q}$

The calculated lattice parameters of the tetragonal crystal structure of the crystals used here were shown in a previous report. ${ }^{10}$ The dimensions of the $z=0.6$ sample were 3.4 $\times 3 \mathrm{~mm}^{2}$ on the $a b$ plane and $1 \mathrm{~mm}$ along the $c$ axis. Measurements of magnetostriction along the $c$ axis were done by means of a conventional strain gauge method at the Tsukuba Magnet Laboratory, the National Institute for Materials Science (NIMS) and at the High Field Laboratory for Superconducting Materials, Institute for Materials Research, Tohoku University. Except for an initial cooldown, the sample was zero-field cooled from $200 \mathrm{~K}$ down to low temperatures. We then started measuring the isothermal magnetostriction upon increasing (or decreasing) the applied fields, parallel to the $c$ axis. Next, warming the sample from low temperatures up to $200 \mathrm{~K}$ in the absence of field and keeping the sample fixed at $200 \mathrm{~K}$ for $2 \mathrm{~h}$, the sample was then cooled down to the low $T$. The typical cooldown time needed was about $2 \mathrm{~h}$. Checking the stability of the sample's temperature, we restarted measuring the magnetostriction. For each measurement, we repeated the same procedure. The normal sweep rate was set to be $0.26 \mathrm{~T} / \mathrm{min}$. Hydrostatic pressures in the magnetostriction experiment were applied by a clamp-type cell using Fluorinert as a pressure-transmitting medium. The pressure was calibrated by the critical temperature of lead. After the magnetostriction measurement under the pressure, we restarted measuring $d L_{c}(H)$ using the same clamp-type cell but without the applied pressure. Next, we examined the $c$-axis magnetostriction as a function of the applied current on the $a b$ plane at ambient pressure. Once the sample was removed from the clamp-type cell, it was set in a conventional cryostat for magnetotransport measurements. On both ends of the sample the electrodes for injection of the electric current were formed using a gold paste and $\mathrm{Cu}$ wires were then attached with silver paste. For magnetoresistance measurements, we carried out the same temperature profile as that in the magnetostriction experiments. 

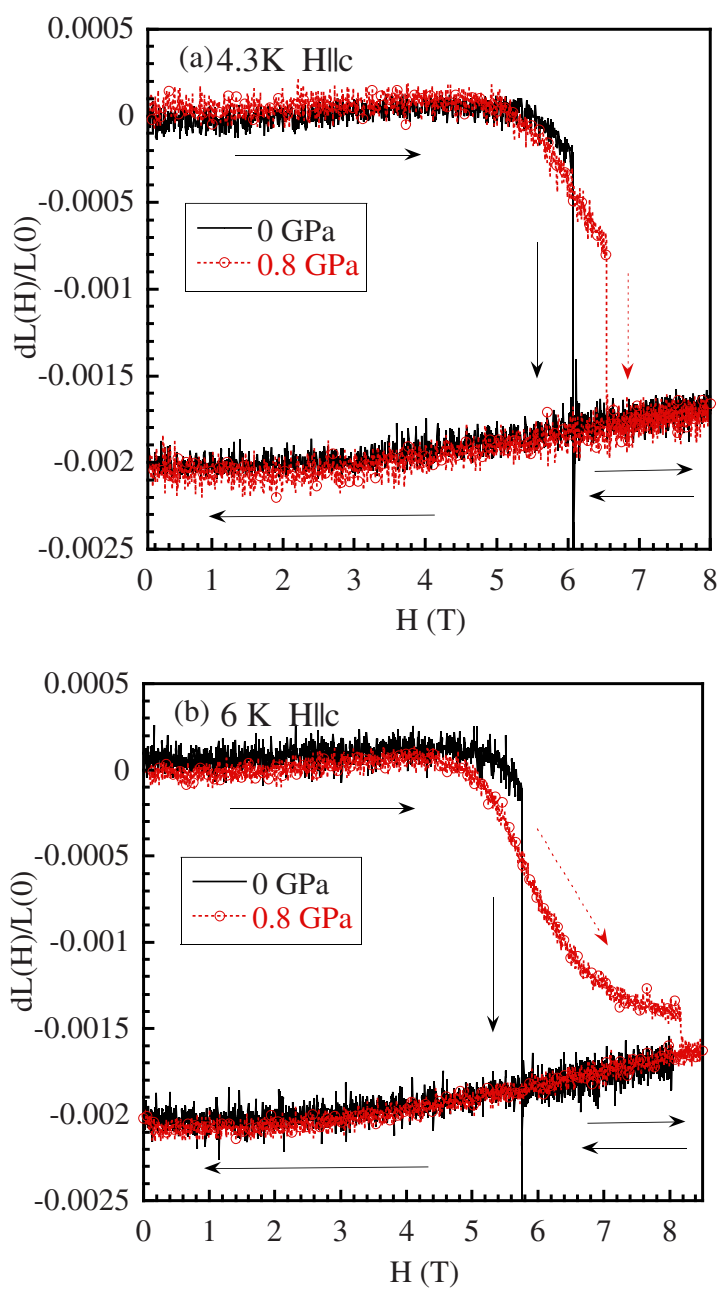

FIG. 1. (Color online) The $c$-axis magnetostriction, $d L_{c}(H) / L_{c}(0)$, of $\left(\mathrm{La}_{0.4} \mathrm{Pr}_{0.6}\right)_{1.2} \mathrm{Sr}_{1.8} \mathrm{Mn}_{2} \mathrm{O}_{7}$, under both ambient pressure and a hydrostatic pressure of $0.8 \mathrm{GPa}$ at low temperatures of (a) 4 and (b) $6 \mathrm{~K}$. The applied field is parallel to the $c$ axis $\left(H \|_{c}\right)$.

\section{RESULTS AND DISCUSSION}

Figure 1 shows the $c$-axis magnetostriction, $d L_{c}(H) / L_{c}(0)$, of $\left(\mathrm{La}_{0.4} \mathrm{Pr}_{0.6}\right)_{1.2} \mathrm{Sr}_{1.8} \mathrm{Mn}_{2} \mathrm{O}_{7}$, under both ambient pressure and a hydrostatic pressure of $0.8 \mathrm{GPa}$ at lower temperatures, where the applied field is parallel to the $c$ axis $(H \| c)$. Here, the value of $d L_{i}(H)$ is defined as $L_{i}(H)-L_{i}(0)$. First of all, the steplike lattice transformations appear at 4 and $6 \mathrm{~K}$ at ambient pressure, as shown in Fig. 1.

On the other hand, the application of external pressure on the crystal substantially suppresses the steplike transition, causing a broad variation in the magnetostriction. At $4.3 \mathrm{~K}$, the critical field $H_{c}$ is increased from $6.1 \mathrm{~T}$ at ambient pressure up to $6.6 \mathrm{~T}$ at $0.8 \mathrm{GPa}$. At $6 \mathrm{~K}$, a huge steplike transition vanishes under the pressure and a continuously smooth variation is observed at high fields, followed by the appearance of a tiny step around $8 \mathrm{~T}$. However, the characteristic field signifying the onset of the field-induced metamagnetic transition, which is not the critical field of the step transitions, is rather lowered at the presence of the applied pressure, as the pressure data measured at high $T$ in Fig. 2 show.

When the magnetic field is applied to the $a b$ plane $(H \| a b)$, we obtain similar results, as shown in Fig. 3. These
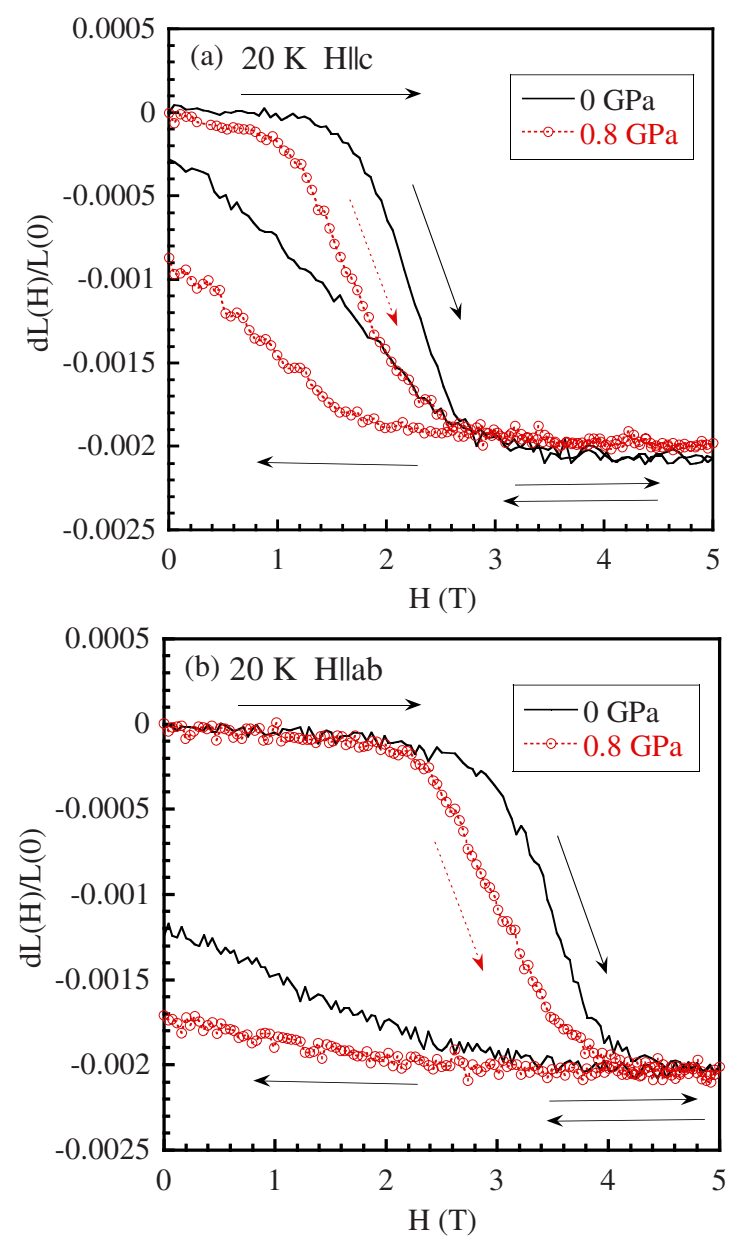

FIG. 2. (Color online) The $c$-axis magnetostriction, $d L_{c}(H) / L_{c}(0)$, of $\left(\mathrm{La}_{0.4} \mathrm{Pr}_{0.6}\right)_{1.2} \mathrm{Sr}_{1.8} \mathrm{Mn}_{2} \mathrm{O}_{7}$, under both ambient pressure and a hydrostatic pressure of $0.8 \mathrm{GPa}$ at $20 \mathrm{~K}$ : (a) $H \|_{c}$ and (b) $H \| a b$.

findings are indicative of the occurrence of steplike transformation almost independent of the easy axis of magnetization. The small differences in the higher critical fields in the case of $H \| a b$ probably arise from the easy axis of $M$ lying along the $c$ axis. Under the application of $0.8 \mathrm{GPa}$, we obtain both $H_{c} \sim 7.6 \mathrm{~T}$ at $4.3 \mathrm{~K}$ and $8.1 \mathrm{~T}$ at $6 \mathrm{~K}$ accompanied by a degradation of a discontinuous jump and the disappearance of any steplike profile in the $d L_{c}(H) / L_{c}(0)$ curve at $8 \mathrm{~K}$.

In our previous paper, ${ }^{12}$ we found out that the application of pressure enhances a field-induced ferromagnetic state of Pr-substituted $\mathrm{La}_{1.2} \mathrm{Sr}_{1.8} \mathrm{Mn}_{2} \mathrm{O}_{7}$. Our findings are understood from the viewpoint that the double-exchange interactiondriven FM state is strengthened by the applied pressure. For the magnetic-field-cooled sample, the remarkable step observed in $d L_{c}(H) / L_{c}(0)$ is monotonically decreased upon increasing the cooling field from 1 up to $1.6 \mathrm{~T}$, accompanied by a nonlinear dependence of $H_{c}{ }^{13}$ The steplike behavior vanishes when the cooling field exceeds $1.6 \mathrm{~T}$. For each fieldcooled run, we expect that the FM region is initially formed within the PMI matrix before the magnetostriction measurements are carried out. A larger volume fraction of the FM phase causes a more suppressed variation in $d L$. Thus, a pressure-induced suppression in the ultrasharp magnetostric- 

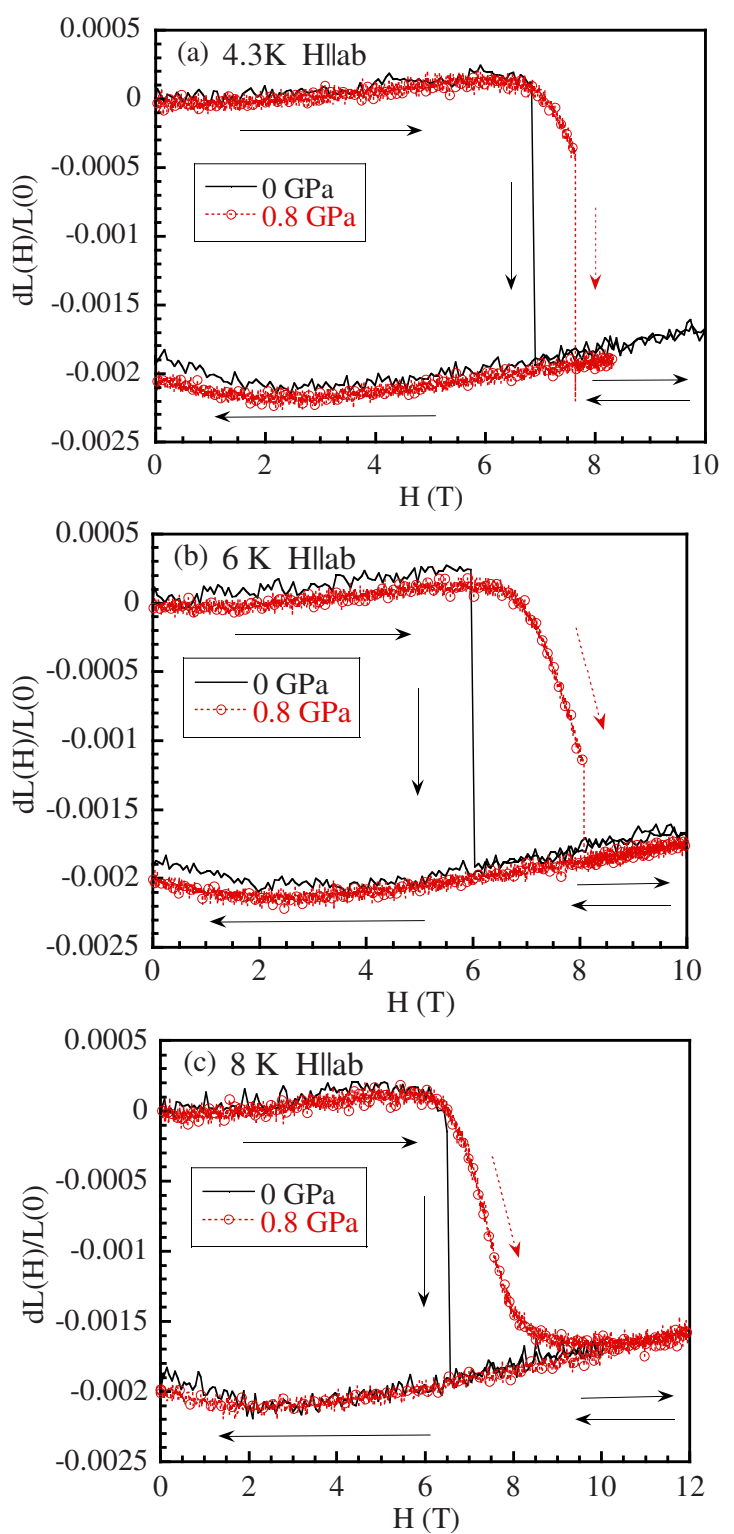

FIG. 3. (Color online) The $c$-axis magnetostriction, $d L_{c}(H) / L_{c}(0)$, of $\left(\mathrm{La}_{0.4} \mathrm{Pr}_{0.6}\right)_{1.2} \mathrm{Sr}_{1.8} \mathrm{Mn}_{2} \mathrm{O}_{7}$, under both ambient pressure and a hydrostatic pressure of $0.8 \mathrm{GPa}$ at low temperatures of (a) 4, (b) 6, and (c) $8 \mathrm{~K}$. The applied field is parallel to the $a b$ plane $(H \| a b)$.

tion is a reasonable result since the applied pressure enhances the FM state.

Next, we carry out the magnetostriction measurement under the applied electric current, to examine a relationship between charge transport and steplike lattice deformation that appeared in CMR manganites. In the case of ambient pressure, the $c$-axis magnetostriction data at $4.3 \mathrm{~K}$ are presented in Fig. 4(a) as a function of excited current $(I=0.01$, 1 , and $2 \mathrm{~mA}$ ). Here, the electric current is applied on the $a b$ plane parallel to the $\mathrm{MnO}_{2}$ double layers. Injection of the electric current over $2 \mathrm{~mA}$ exhibits no discontinuous profile in $d L_{c}(H) / L_{c}(0)$. We note that the bath temperature is very stable against the applied current up to $2 \mathrm{~mA}$ within $\sim 0.5 \%$ and Joule heating of the sample affects no contribution to the present behavior.
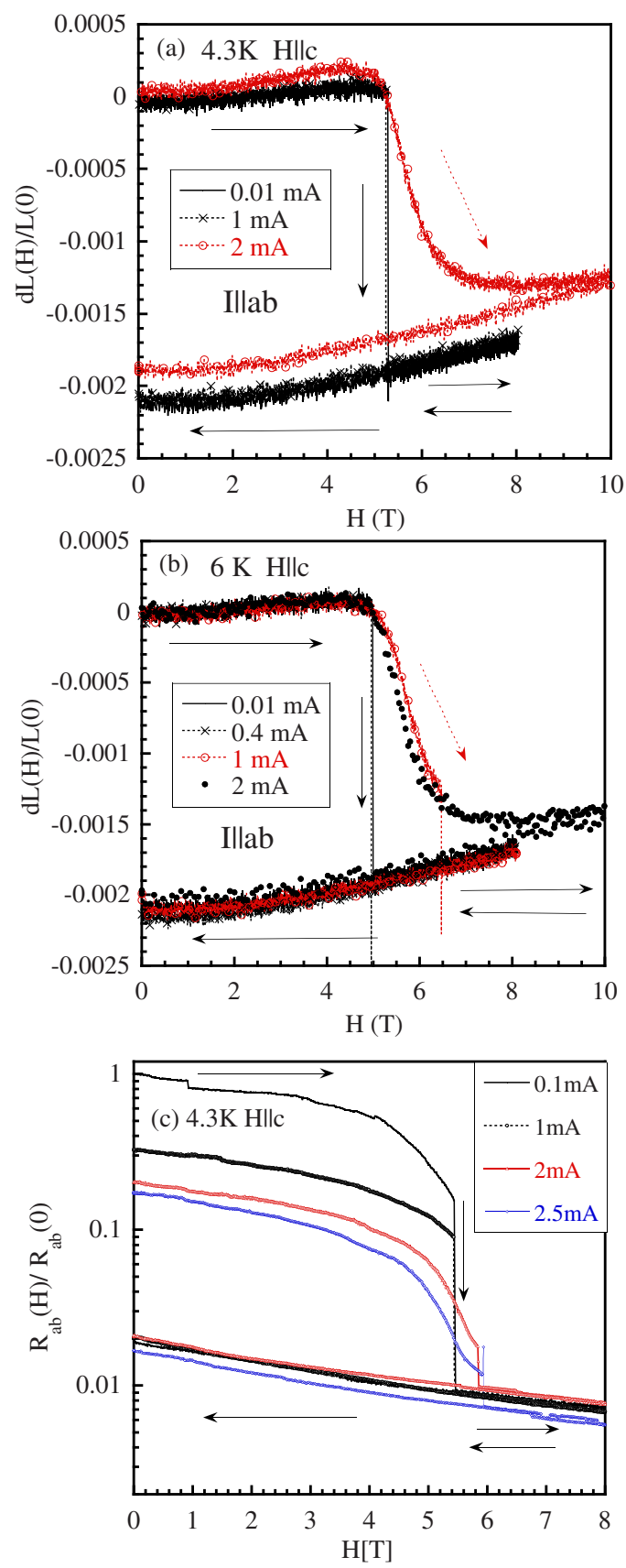

FIG. 4. (Color online) The $c$-axis magnetostriction, $d L_{c}(H) / L_{c}(0)$, of $\left(\operatorname{La}_{0.4} \operatorname{Pr}_{0.6}\right)_{1.2} \mathrm{Sr}_{1.8} \mathrm{Mn}_{2} \mathrm{O}_{7}$, under the electric current applied on the $a b$ plane at low temperatures of (a) 4 and (b) 6 $\mathrm{K}$. The applied field is parallel to the $c$ axis. (c) The $a b$-plane magnetoresistance $R_{a b}$ of the $z=0.6$ sample at $4.3 \mathrm{~K}$ as a function of the applied current from 0.1 up to $2.5 \mathrm{~mA}$. The $R_{a b}(\mathrm{H})$ data are normalized by $R_{a b}(0)$, which is the zero-field resistance at the lowest applied current $(0.1 \mathrm{~mA}) ;(H \| c)$.

For comparison, let us now display in Fig. 4(c) the $a b$-plane magnetoresistance $R_{a b}$ of the $z=0.6$ sample as a function of the applied current from 0.1 up to $2.5 \mathrm{~mA}$. First of all, the application of higher current gives rise to a degradation of the magnetoresistive steps observed at lower currents, which is almost consistent with the magnetostriction data shown in Fig. 4(a). Next, the zero-field resistance mea- 
sured at $2 \mathrm{~mA}$ shows a substantial drop of $\sim 80 \%$, in comparison to $R_{a b}(0)$ at the lowest current $(0.1 \mathrm{~mA})$. This result indicates the current-induced destabilization of the PMI matrix, although the physical role of the application on an electric field is not clear. Similar results are reported in a systematic study on nonlinear transport of a non-charge-ordered manganite $\operatorname{Pr}_{0.8} \mathrm{Ca}_{0.2} \mathrm{MnO}_{3}$, which is not so far from the $\mathrm{COO}$ region. ${ }^{14}$ In a lightly Pr-substituted crystal of $\left(\mathrm{La}_{0.8} \mathrm{Pr}_{0.2}\right)_{1.2} \mathrm{Sr}_{1.8} \mathrm{Mn}_{2} \mathrm{O}_{7}$ with $T_{c} \sim 90 \mathrm{~K}$, we also have obtained a current-induced remarkable drop in the $a b$-plane resistance at selected temperatures just above $T_{c}$ (not shown here). The formation of short-range charge ordering in the paramagnetic phase of an optimally doped parent crystal $\mathrm{La}_{1.2} \mathrm{Sr}_{1.8} \mathrm{Mn}_{2} \mathrm{O}_{7}$ has been observed in a previous study. ${ }^{15} \mathrm{Ac}-$ cordingly, we expect that the application of the electric current removes the localized carriers, such as the $\mathrm{COO}$ insulating clusters, from the PMI phase of the Pr-substituted crystal, causing a substantial reduction in the zero-field resistance at higher currents.

Furthermore, neutron-scattering measurements on a bilayered manganite $\mathrm{La}_{2-2 x} \mathrm{Sr}_{1+2 x} \mathrm{Mn}_{2} \mathrm{O}_{7}(x=0.38)$ revealed that the CE-type (zig-zag FM chains with antiferromagnetic interchain coupling) $\mathrm{COO}$ clusters freeze upon decreasing temperature from $T \sim 310 \mathrm{~K}$, preventing the formation of a long-range $\mathrm{COO}$ state. ${ }^{16}$ The authors also argued that the PMI state of the crystal without Pr substitution is caused by an orbital frustration and stabilized down to the FMM transition temperature $T_{c} \sim 114 \mathrm{~K}$. For the present Pr-substituted sample, one believes that the short-range COO clusters accompanied by the orbital frustration are present within the PMI matrix. As discussed in Ref. 13, we suppose that the orbital frustration has some relationship with the critical instability of the metastable state of the free energy, resulting in the steplike phase transition. The application of hydro- static pressure stabilizes the ferromagnetic interaction within the paramagnetic matrix and the applied current reinforces an itinerant state of charge carriers within the insulating matrix. In other words, these external variables probably lead to a collapse of the CE-type antiferromagnetic charge-ordered clusters if clusters of this type are distributed within the sample. In addition, Mahendiran et $a .^{3}$ claimed that the charge-orbital-ordered domains in the Co-doped $\mathrm{Pr}_{0.5} \mathrm{Ca}_{0.5} \mathrm{MnO}_{3}$ are smaller than those in the undoped $\operatorname{Pr}_{0.7} \mathrm{Ca}_{0.3} \mathrm{MnO}_{3}$, suggesting that the onset temperature of the step transitions in the Co-doped compound is higher than that in the undoped one. We emphasize that the distribution of short-range COO clusters embedded in the matrix provides a significant clue for our understanding of the steplike transformations in CMR manganites. The ultrasharp PMIFMM transition induced by the magnetic field at low temperatures is in its origin quite different from the standard insulator-metal transition associated with the metamagnetic transition at higher $T$.

In summary, we have demonstrated the influence of pressure on the steplike lattice deformation of single crystalline bilayered manganite $\left(\mathrm{La}_{0.4} \mathrm{Pr}_{0.6}\right)_{1.2} \mathrm{Sr}_{1.8} \mathrm{Mn}_{2} \mathrm{O}_{7}$. The external perturbations such as the application of pressure and electric current give a substantial suppression on the steplike transition, resulting in a broad change in the magnetostriction. It is a future problem to resolve what role the COO clusters within the PMI phase play in the occurrence of the steplike phenomenon observed.

\section{ACKNOWLEDGMENTS}

This work was supported by a Grant-in-Aid for Scientific Research from the Japan Society of the Promotion of Science.

\footnotetext{
*matsukawa@iwate-u.ac.jp

${ }^{1}$ Colossal Magnetoresistive Oxides, edited by Y. Tokura (Gordon and Breach, New York, 2000).

${ }^{2}$ E. Dagotto, Nanoscale Phase Separation and Colossal Magnetoresistance (Springer, New York, 2003).

${ }^{3}$ R. Mahendiran, A. Maignan, S. Hebert, C. Martin, M. Hervieu, B. Raveau, J. F. Mitchell, and P. Schiffer, Phys. Rev. Lett. 89, 286602 (2002).

${ }^{4}$ V. Hardy, A. Maignan, S. Hebert, C. Yaicle, C. Martin, M. Hervieu, M. R. Lees, G. Rowlands, D. M. Paul, and B. Raveau, Phys. Rev. B 68, 220402(R) (2003).

${ }^{5}$ L. M. Fisher, A. V. Kalinov, I. F. Voloshin, N. A. Babushkina, D. I. Khomskii, Y. Zhang, and T. T. M. Palstra, Phys. Rev. B 70, 212411 (2004).

${ }^{6}$ L. Ghivelder, R. S. Freitas, M. G. das Virgens, H. Martinho, L. Granja, G. Leyva, P. Levy, and F. Parisi, Phys. Rev. B 69, 214414 (2004).

${ }^{7}$ Sunil Nair, A. K. Nigam, A. V. Narlikar, D. Prabhakaran, and A. Boothroyd, Phys. Rev. B 74, 132407 (2006).

${ }^{8}$ Da-qian Liao, Young Sun, Ren-fu Yang, Qing-an Li, and Zhaohua Cheng, Phys. Rev. B 74, 174434 (2006).

${ }^{9}$ Y. Moritomo, Y. Maruyama, T. Akimoto, and A. Nakamura,
}

Phys. Rev. B 56, R7057 (1997).

${ }^{10}$ M. Apostu, R. Suryanarayanan, A. Revcolevschi, H. Ogasawara, M. Matsukawa, M. Yoshizawa, and N. Kobayashi, Phys. Rev. B 64, 012407 (2001).

${ }^{11}$ M. Matsukawa, K. Akasaka, H. Noto, R. Suryanarayanan, S. Nimori, M. Apostu, A. Revcolevschi, and N. Kobayashi, Phys. Rev. B 72, 064412 (2005).

${ }^{12}$ M. Matsukawa, A. Tamura, S. Nimori, R. Suryanarayanan, T. Kumagai, Y. Nakanishi, M. Apostu, A. Revcolevschi, K. Koyama, and N. Kobayashi, Phys. Rev. B 75, 014427 (2007).

${ }^{13}$ M. Matsukawa, Y. Yamato, T. Kumagai, A. Tamura, R. Suryanarayanan, S. Nimori, M. Apostu, A. Revcolevschi, K. Koyama, and N. Kobayashi, Phys. Rev. Lett. 98, 267204 (2007).

${ }^{14}$ S. Mercone, A. Wahl, Ch. Simon, and C. Martin, Phys. Rev. B 65, 214428 (2002).

${ }^{15}$ L. Vasiliu-Doloc, S. Rosenkranz, R. Osborn, S. K. Sinha, J. W. Lynn, J. Mesot, O. H. Seeck, G. Preosti, A. J. Fedro, and J. F. Mitchell, Phys. Rev. Lett. 83, 4393 (1999).

${ }^{16}$ D. N. Argyriou, J. W. Lynn, R. Osborn, B. Campbell, J. F. Mitchell, U. Ruett, H. N. Bordallo, A. Wildes, and C. D. Ling, Phys. Rev. Lett. 89, 036401 (2002). 